\title{
Valorisation par des jeunes bovins de deux variétés de maïs de digestibilité différente
}

\author{
P Haurez 1, A Joulie 2, B Carpentier 3 \\ IInstitut de l'Elevage, 14 avenue Joxé, BP 646, 49006 Angers Cedex 01 ; 2 Chambre d'Agriculture de Vendée, \\ Ferme des Etablières, 85013 La Roche sur Yon Cedex; ${ }^{3}$ Association Générale de Producteurs de Maïs, \\ Rue Frère Gagne, BP 463, 60021 Beauvais Cedex, France
}

La prévision de la valeur énergétique du maïs ensilage nécessite de déterminer le coefficient d'utilisation digestive de la matière organique in vivo sur moutons "standard". En laboratoire, pour les mesures de routine, cette estimation passe par le dosage de constituants chimiques, la mesure de la solubilité enzymatique in vitro (Aufrère et MichaletDoreau, 1988, Anim Feed Sci Technol, 20, 203-218) ou la spectroscopie dans le proche infra rouge. Des animaux à fort niveau de production montrent-ils des performances zootechniques différentes sur des maïs dont la digestibilité prédite est différente ? Un programme d'essais sur taurillons et vaches laitières a été réalisé dans ce sens avec la collaboration de SEPROMA (Syndicat des producteurs de semences de maï). Les essais conduits sur jeunes bovins dans la ferme expérimentale des Etablières en Vendée sont présentés ici.

Dans deux essais, deux hybrides de précocité voisine ( $A$ et $X$ dans essai $1, A$ et $B$ dans essai 2), cultivés et récoltés de façon identique mais différents par au moins 2 points de digestibilité de la matière organique (DMO) mesurée sur moutons (méthode in vivo), ont été distribués à deux lots de 26 et 22 jeunes bovins charolais. Par rapport au maïs $A$, les maïs $X$ et $B$ se caractérisaient par un taux moyen de MS supérieur de 4,6 points, une teneur en $C B$ comparable (maïs $A=18,2 \%$ - maïs $X$ et $B=19,2 \%$ ), une teneur en MAT respectivement inférieure de 12 et $15 \mathrm{grammes} / \mathrm{kg}$ de MS. L'amidon était plus variable : $-19 \mathrm{~g} / \mathrm{kg}$ de MS pour le maïs $X$ et $+26 \mathrm{~g}$ pour le maïs $B$ par rapport à $A$.

Le maïs $A$ a présenté une solubilité enzymatique moyenne de 66,6 points. Celle des maïs $X$ et $B$ était respectivement inférieure de 3,9 et 2 points, se traduisant ainsi par une valeur en UFV calculée à partir d'un modèle intégrant cette mesure de $-0,06$ UFV $(-8 \%)$ pour le maïs $X$ et $-0,04$ UFV $(-5 \%)$ pour le maïs $\mathrm{B}$.

Les différents maīs ont été distribués ad libitum à des jeunes bovins d'un poids vif inital moyen de $387 \mathrm{~kg}$. Ils recevaient la même complémentation jounalière : $\mathrm{t}$ soja $=$ $1,3 \mathrm{~kg}$, céréales $=0,6 \mathrm{~kg}, \mathrm{CMV}=0,18 \mathrm{~kg}$, foin $=0,25 \mathrm{~kg}$.

Dans les deux essais, la consommation de mais ensilage est comparable mais les croissances des lots recevant les maïs à plus faible digestibilité sont significativement inférieures (essai $1:-152 \mathrm{~g} /$ jour - essai $2:-81$ $\mathrm{g} /$ jour). A partir des performances exprimées par les animaux, les valeurs énergétiques des mais $X$ et $B$ apparaissent respectivement inférieures de 10 et $7 \%$ à celle du maïs $A$.

En conclusion, la consommation du maïs à plus faible digestibilité (DMO mesurée in vivo) s'est traduit par une baisse de la croissance des jeunes bovins. La valorisation du maïs par les animaux correspond assez bien aux valeurs du maïs prédites à partir de la mesure de solubilité enzymatique selon la méthode Aufrère. Cependant, ces résultats demandent à être confirmés.

\begin{tabular}{lcccc} 
& \multicolumn{2}{c}{ essai 1 } & \multicolumn{2}{c}{ essai 2 } \\
& A & $X$ & A & B \\
Durée essai (jours) & 186 & 186 & 160 & 160 \\
MS totale ingérée $(\mathrm{kg} / \mathrm{j})$ & 9,08 & 9,04 & 8,78 & 8,93 \\
Croissance $(\mathrm{g} / \mathrm{j})$ & $1499^{\star *}$ & $1347^{* *}$ & $1481^{*}$ & $1400^{\star}$ \\
MS/kg gain poids vif $(\mathrm{kg})$ & 6,06 & 6,71 & 5,93 & 6,38
\end{tabular}

\footnotetext{
${ }^{\star \star}$ différence significative au seuil de $1 \%$; * au seuil de $5 \%$
} 